\title{
Jeramana, voisine de Damas : le devenir d'une ville réceptacle de réfugiés irakiens
}

Jeramana, next of Damascus: the future of a city receptacle of iraqi refugees

\section{Cyril Roussel}

\section{OpenEdition}

\section{Journals}

Édition électronique

URL : http://journals.openedition.org/rge/2041

DOI : $10.4000 /$ rge.2041

ISSN : $2108-6478$

Éditeur

Association des géographes de l'Est

Édition imprimée

Date de publication : 31 décembre 2009

ISSN : 0035-3213

Référence électronique

Cyril Roussel, « Jeramana, voisine de Damas : le devenir d'une ville réceptacle de réfugiés irakiens », Revue Géographique de l'Est [En ligne], vol. 49 / 4 | 2009, mis en ligne le 01 novembre 2010, consulté le 08 septembre 2020. URL : http://journals.openedition.org/rge/2041 ; DOI : https://doi.org/10.4000/ rge.2041

Ce document a été généré automatiquement le 8 septembre 2020

Tous droits réservés 


\title{
Jeramana, voisine de Damas : le devenir d'une ville réceptacle de réfugiés irakiens
}

\author{
Jeramana, next of Damascus: the future of a city receptacle of iraqi refugees
}

\author{
Cyril Roussel
}

1 L'étude du phénomène migratoire en Syrie montre l'existence d'une double dynamique. La première est spatiale ${ }^{1}$ : Damas reçoit une part écrasante des flux migratoires du pays qui convergent surtout vers ses périphéries, puisque ce sont les banlieues, communes agglomérées à la capitale ou bien celles des couronnes concentriques, qui absorbent la plupart des migrants issus de mouvements tant internes qu'externes. La seconde est culturelle : les itinéraires migratoires conduisent généralement les migrants vers des centralités urbaines qui offrent certes des opportunités économiques (services, emplois) mais qui garantissent surtout la proximité socio-culturelle ${ }^{2}$.

2 Ces mouvements de populations dirigés vers certaines périphéries de Damas, terminus migratoires des populations issues des quatre coins du pays et des Etats voisins en conflit et qui répondent en grande partie à des dynamiques et stratégies d'implantation communautaire, semblent générer une organisation socio-spatiale originale. D’un côté, une forte différenciation spatiale se produit entre les banlieues dynamiques qui gagnent de la population et celles qui stagnent; de l'autre, cette dynamique est à l'origine d'atmosphères urbaines fortement distinctes d'une banlieue à une autre. Pour mieux approcher cette dynamique organisationnelle de l'espace urbain en œuvre dans le Grand Damas, nous proposons de nous appuyer sur un exemple emblématique : celui de la ville de Jeramana.

3 Jeramana offre un exemple particulièrement stimulant de ville qui doit son développement tant spatial qu'économique à l'afflux de populations migrantes ${ }^{3}$. Au cœur des crises régionales (conflit israélo-arabe, conflit libanais, conflit irakien) et des transformations internes à la Syrie (renforcement du rôle de Damas et exode rural), une ville comme Jeramana est devenue un réceptacle où se concentrent divers types de populations migrantes. Son originalité réside dans le fait qu'elle polarise depuis 
longtemps des flux de migrants syriens issus de groupes confessionnels minoritaires (Druzes, Chrétiens) et plus récemment des réfugiés irakiens eux aussi appartenant à des minorités confessionnelles (Chrétiens, Sabéens).

4 L'analyse de la genèse d'un tel territoire urbain, que s'approprient des membres issus de minorités confessionnelles, devrait nous permettre de comprendre le rôle qu'a joué Jeramana pour toute une partie des réfugiés irakiens, tant dans leur choix d'implantation que dans leurs stratégies migratoires plus globales. Nous chercherons aussi à savoir en quoi cette ville constitue un espace de transit, ou bien un refuge durable pour une partie des Irakiens en Syrie. La durabilité, s'il y a, de l'installation d'un grand nombre de migrants irakiens dans une ville moyenne pose la question de leur capacité à s'insérer dans l'économie locale et à transformer, par leur présence, leurs investissements et leurs initiatives, l'espace même de la ville.

Ce travail s'appuie sur une série d'entretiens que nous avons menés entre 1998 et 2009, c'est-à-dire avant et après le déclenchement du conflit irakien en 2003. Nous avons ainsi suivi l'évolution du développement urbain de cette banlieue de Damas sur une période assez longue mais aussi observé, au fil des années, sa recomposition sociale et spatiale en lien avec l'afflux de réfugiés irakiens. Conscient des limites inhérentes au traitement de l'appareil statistique produit tant par les agences de l'ONU (UNHCR notamment) que par les autorités syriennes (locales et nationales), nous avons privilégié l'analyse de terrain en multipliant les interviews de notables locaux, d'intellectuels (chefs religieux, responsables politiques, enseignants, écrivains), de simples habitants de Jeramana; de membres d'ONG mais aussi de diverses catégories de réfugiés irakiens (étudiants, commerçants, employés d'ONG, entrepreneurs).

\section{La ville de Jeramana : une croissance liée aux mouvements migratoires à caractère communautaire}

\section{A. Jeramana : un réceptacle migratoire}

Dans les années 1960, Jeramana n'est qu'une modeste bourgade située à une dizaine de kilomètres au Sud-Ouest de la capitale du jeune Etat, Damas. Avec à peine 6500 habitants, elle constitue alors un petit centre agricole dans une zone riche en arbres fruitiers, la Ghouta de Damas. Quarante ans plus tard, sa population a été multipliée par vingt. Son taux moyen de croissance annuelle sur la période 1960-2004 a flirté avec les 7 $\%$ contre un peu plus de $3 \%$ pour l'ensemble de la population syrienne. Entre 1976 et les années 2000, la superficie de la municipalité de Jeramana a quadruplé passant de 115 hectares à $450^{4}$.

7 Ainsi en 2004, Jeramana est devenue une ville moyenne de près de 120000 habitants ${ }^{5}$ (réfugiés irakiens exclus) mais dans laquelle seulement 24200 habitants sont inscrits sur les registres de l'Etat civil, soit tout juste $20 \%$ de sa population. Parmi ces inscrits, 5 700 ont transféré leur registre d'une autre commune à Jeramana dans les années 1990 (surtout des Chrétiens) et ne sont donc pas natifs de cette ville. Compte tenu de cette précision, plus de $85 \%$ de la population qui réside dans la ville sont issus de l'émigration interne.

8 Depuis le déclenchement du conflit irakien au début de l'année 2003, l'arrivée d'abord progressive puis massive (entre 2004 et 2008) de réfugiés irakiens a provoqué une 
explosion démographique sans précédent. Si la municipalité avance le chiffre de 163 000 ressortissants irakiens début 2008, le nombre d'inscrits au HCR n'était pourtant que de 59000 personnes $^{6}$. Il semble raisonnable de parler d'un quasi doublement de la population urbaine en quelques années et ce avant 2008, année du retour de nombreux réfugiés en Irak. On note, durant cette période, une densification plus qu'un étalement urbain à Jeramana; la ville se développe à la verticale.

Figure 1 : La rue principale de Jeramana en 2008 : immeuble en construction à côté d'une des dernières habitations basses de la ville

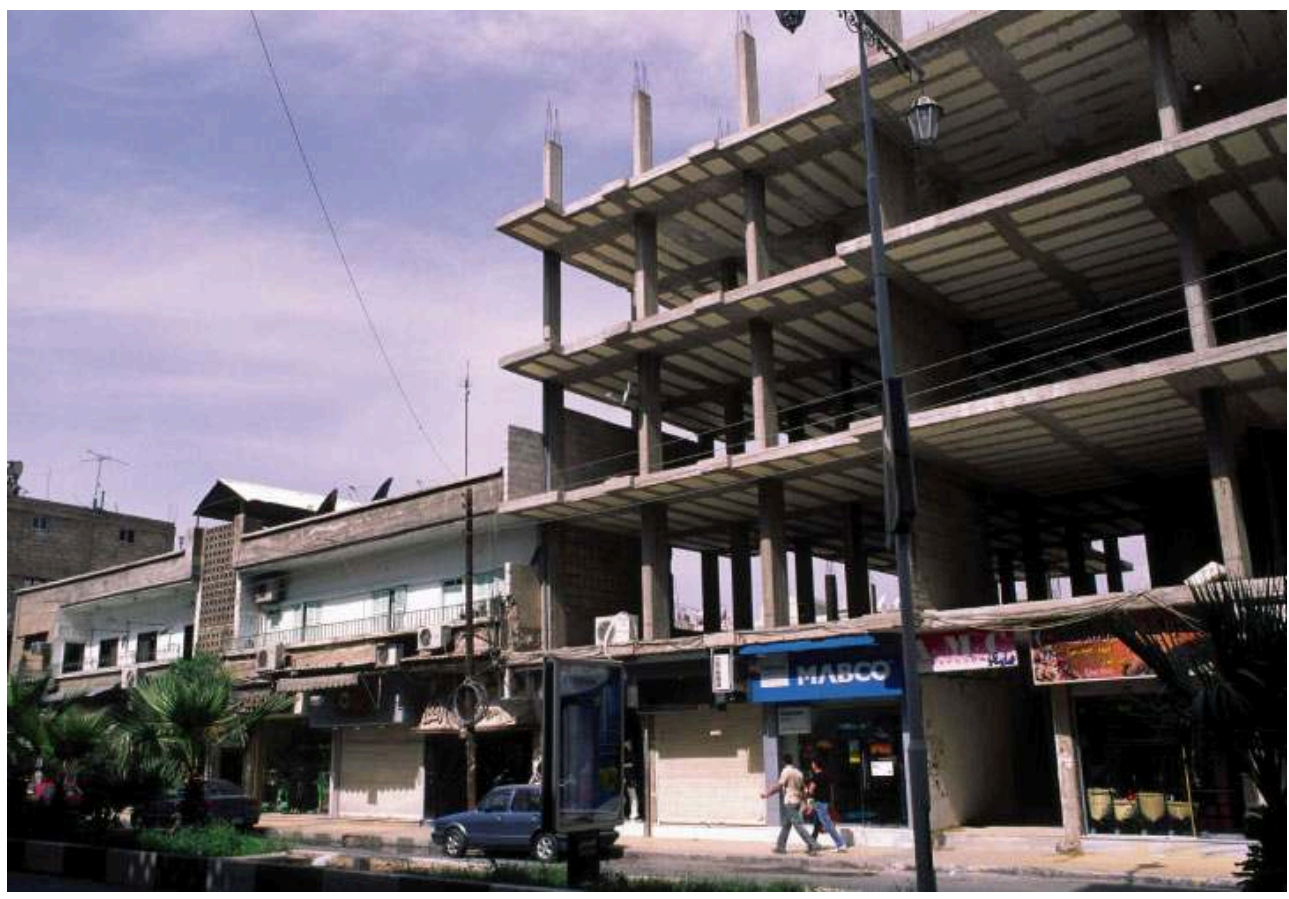

Cliché : Cyril Roussel, août 2008

Pour mieux comprendre le rôle de Jeramana dans l'espace migratoire des diverses populations migrantes qui s'y sont établies, il semble primordial de revenir sur l'analyse des réseaux migratoires propres à chaque groupe de migrants. Ainsi, nous serons à même de comprendre comment Jeramana et plus généralement certaines villes de la banlieue damascène sont devenues de véritables territoires appropriés par des communautés religieuses.

\section{B. Jeramana, premier pôle migratoire des Druzes de Syrie}

10 En fonction de leur appartenance confessionnelle (druze, chrétienne ou sunnite), les migrants ont privilégié certains pôles particuliers d'installation au sein de l'agglomération damascène favorisant ainsi des regroupements communautaires. La croissance de la ville de Jeramana, village druze à l'origine, est liée directement à l'émigration druze en provenance des montagnes voisines, alors que la part d'autres migrants est comparativement très réduite. Mais Jeramana n'est pas pour autant l'unique réceptacle de l'exode rural druze.

11 Dans les années 1960-70, les mohafaza-s de Daraa et de Sweida (les deux provinces méridionales les plus peuplées) fournissent une part importante des migrants vers les 
banlieues de Damas. Par rapport à la population globale respective de chaque mohafaza, Sweida et Daraa sont les deux gouvernorats de Syrie qui ont contribué le plus, au cours de ces décennies, aux échanges internes avec l'agglomération de Damas, exception faite de Quneitra ${ }^{7}$. Une analyse en valeurs relatives permet de vérifier que le gouvernorat de Sweida (à $90 \%$ druze) est en tête des régions « déversoirs " vers Damas. D'après les données du recensement de 1960, environ un Druze sur vingt de la province de Sweida, avait déjà choisi de s'installer dans la Ghouta à cette époque, contre seulement un Sunnite du Hauran sur quatre-vingts. Cette concentration relative des Druzes de Sweida dans la Ghouta a une explication communautaire. Quelques communes de la Ghouta, comme Jeramana, Achrafiyeh et Sahnaya, Jdaidat Artous, servent de réceptacles aux Druzes qui profitent, pour leurs migrations, de la présence de communautés localement établies depuis des siècles. Jeramana est le principal pôle d'établissement des migrants druzes de Syrie dans la périphérie de Damas, affirmant ainsi son rôle de terminus communautaire dans le processus migratoire interne des Druzes. Dans les années 80 et 90, l'immigration druze à Jeramana et les autres terminus communautaires s'est poursuivie, mais aucune statistique n'est disponible.

En ce qui concerne le reste de la communauté druze (établie dans le Djebel Shaykh à la frontière syro-libanaise et dans le Djebel Soumak ${ }^{8}$, au Nord de la Syrie - mohafaza d'Idlib), les migrants ont eux aussi orienté leur mouvement d'émigration interne vers les quelques "communes druzes" de l'agglomération damascène. Nos enquêtes, réalisées entre 2002 et 2005 dans leurs villages de départ, montrent qu'une forte majorité des émigrés druzes à l'intérieur de la Syrie s'est établie dans la commune de Jeramana depuis les années 1960. La primauté des réseaux communautaires et familiaux dans la structuration de la mobilité des Druzes en Syrie a généré des regroupements confessionnels dont Jeramana illustre le processus.

\section{Une ville devenue attractive pour d'autres minorités confessionnelles : Chrétiens syriens, Chrétiens irakiens, Sabéens irakiens}

13 Dans les années 1980 et 1990, de nombreuses familles chrétiennes de Damas quittèrent les quartiers centraux de Bab Touma, Qassa et Tijara, pour s'installer en périphérie de la capitale. Ils furent rapidement rejoints, à la même période, par des Chrétiens d'origine rurale issus de villages des quatre coins du pays - gouvernorats de Sweida, Daraa, Homs, comme ceux du wadi Nazara, mais aussi de Mechta el Helou, Kafroun, et des provinces orientales : Hassake, Qamishli...

14 Le mode de vie des Druzes (femmes non voilées, absence de prosélytisme religieux, repli des religieux dans la spiritualité) et l'atmosphère plus "libre » de Jeramana (mixité homme-femme dans l'espace public, nombreux lieux de sociabilité, consommation d'alcool) a favorisé l'établissement de populations chrétiennes dans cette commune de la Ghouta plus particulièrement. Ils représentent entre 10 et $20 \%$ de l'effectif démographique de la ville avant l'arrivée des Irakiens.

15 L'installation des Chrétiens répond à des stratégies résidentielles qui rendent leur implantation à Jeramana relativement pérenne, tout comme pour les Druzes. Au milieu des années 1980, la Syrie a vécu une grave crise économique qui s'est traduite entre autre par l'augmentation des prix des loyers à Damas. De nombreux chrétiens de Damas-ville ont alors, dans les années qui suivirent, revendu leurs biens immobiliers 
pour racheter un appartement à Jeramana alors 5 à 6 fois moins cher et réaliser ainsi une bonne opération financière. La ville de Jeramana ne possède cependant pas de lieu de culte chrétien car l'implantation de ces populations est récente ; ainsi, les Chrétiens de Jeramana se rendent-ils à Damas pour pratiquer leur culte.

Depuis l'offensive américaine en Irak, lancée dès 2003, plusieurs dizaines de milliers de réfugiés ont privilégié Jeramana comme lieu d'installation. Elle est devenue un pôle de concentration des Irakiens de confession chrétienne et sabéenne ${ }^{9}$. Comme l'indiquent G. Chatelard et K. Doraï (2009), «certaines minorités comme les chrétiens ou les mandéens sont surreprésentés dans le groupe des réfugiés enregistrés auprès du HCR (...) ». Ainsi, on peut observer, pour l'année 2008, que près de $15 \%$ des réfugiés irakiens en Syrie sont de confession chrétienne ${ }^{10}$ et que $4 \%$ sont de confession mandéenne. En Irak, avant le déclenchement du conflit dans les années 1990, ils représentaient respectivement environ $5 \%$ et $0,25 \%$ de la population irakienne dans le pays (voir figure 2). Les plus nombreux sont les sunnites avec près de $58 \%$ du total alors qu'ils ne représentent qu'environ $40 \%$ des effectifs dans leur pays.

Figure 2 : Comparaison entre la répartition par confession de la population irakienne en Irak (avant 2003) et la répartition par confession de la population irakienne réfugiée en Syrie (2008)

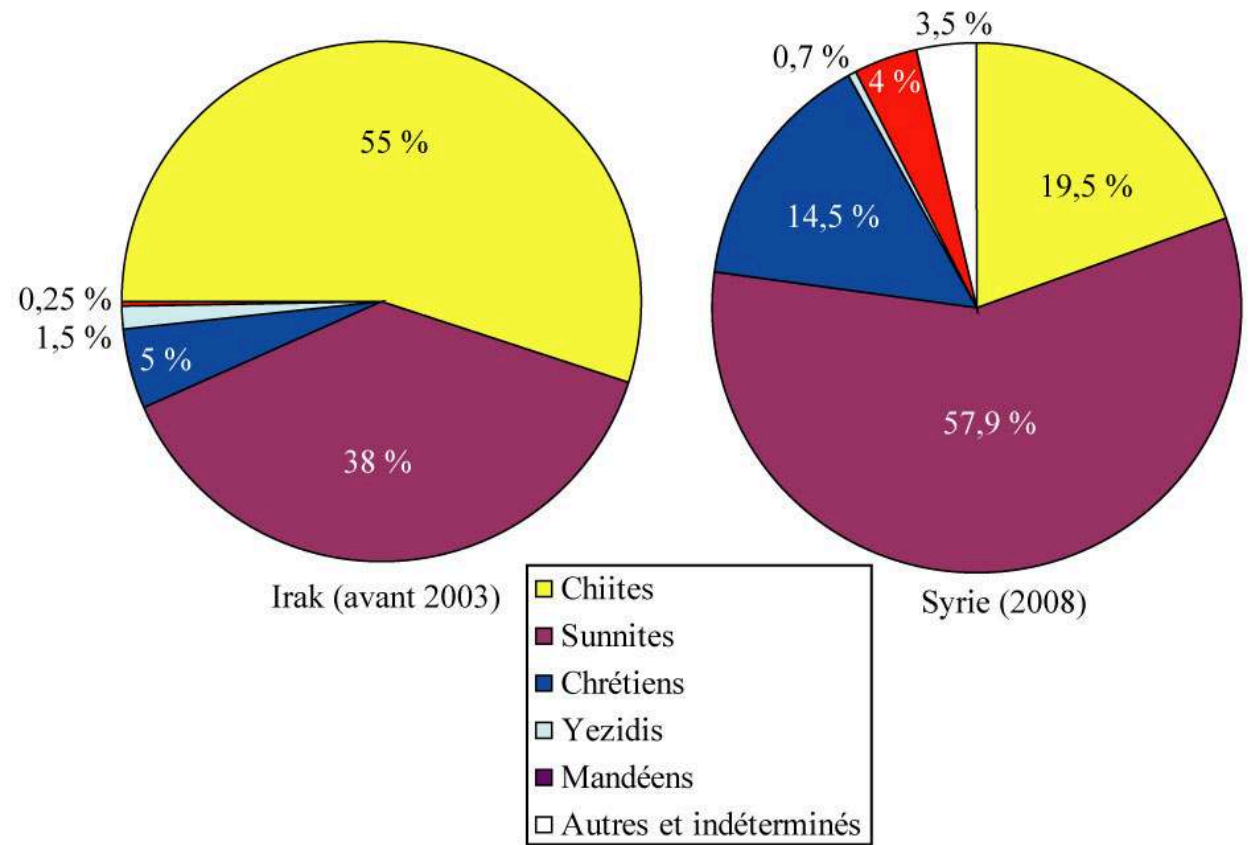

Sources : UNHCR Syria - report on registered Iraqis (02 august 2008)

S'il semble avéré que le coût peu élevé de l'hébergement a été une des raisons de l'installation d'Irakiens dans tel ou tel quartier (Chatelard \& Doraï, 2009), elle n'explique pas les fortes concentrations confessionnelles observables dans certaines villes de la région damascène. En effet, Jeramana ne constitue pas, loin s'en faut, l'unique commune de la banlieue damascène où les loyers sont bas. Par exemple, Akraba ou Mleiha, toutes deux situées à quelques kilomètres de Jeramana, affichent des loyers tout aussi attractifs : mais ces villes sont sunnites et elles n'ont pas attiré les Irakiens de confession chrétienne ou sabéenne. Le contexte socio-culturel a ainsi joué un rôle majeur dans la répartition et la polarisation des Irakiens dans les banlieues du Grand Damas. 
18 A Jeramana, la raison du choix de leur implantation renvoie d'abord à une motivation culturelle et religieuse : la présence des communautés druzes et chrétiennes syriennes est couramment évoquée par les réfugiés irakiens comme motif d'installation dans cette ville. Les entretiens que nous avons conduits dans cette ville auprès d'Irakiens de confession chrétienne ou sabéenne sont unanimes. En voici un extrait emblématique :

"Avant la chute de Saddam Hussein certains d'entre nous connaissaient déjà Jeramana. On savait que l'atmosphère de la ville, peuplée de Druzes et de Chrétiens, permettrait de s'y installer. Au début de la guerre [celle de 2003], nous avons été rapidement menacés et persécutés. Sous le régime de Saddam, nous étions protégés. Les premiers à quitter l'Irak sont donc venus s'installer ici. Ensuite, beaucoup d'autres les ont suivis car notre vie était menacée en Irak. On tente de nous convertir de force ou de nous chasser » (Nazar, commerçant sabéen, Jeramana, 5 octobre 2008).

D'abord envisagé par les réfugiés irakiens eux-mêmes comme provisoire, le séjour syrien se prolonge pour certains d'entre eux. Après sept années de conflit, des segments entiers de la société irakienne semblent ne plus être en mesure de franchir la frontière dans le sens inverse, comme nous allons le voir.

\section{L'attente incertaine d'une partie des Irakiens dans le refuge syrien}

Depuis le début du conflit irakien, la Syrie est devenue le pays du Moyen-Orient qui accueille le plus grand nombre de migrants irakiens. Cependant, leur présence varie fortement en fonction de la politique aux frontières mise en place par les autorités syriennes. Pour les réfugiés en Syrie qui se sentent menacés en Irak, l'attente dans le refuge syrien semble se prolonger, leur présence se pérenniser.

\section{A. Les phases de la migration irakienne :}

La présence irakienne en Syrie remonte aux années 1970 (Kawakibi, 2008 ; Doraï, 2009) Il s'agissait pour la plupart de réfugiés politiques qui fuyaient la répression du régime de Saddam Hussein ou le service militaire. Si le HCR estimait qu'en 2003 entre 60000 et 70000 Irakiens résidaient en Syrie, la plupart n'étaient pas enregistrés comme réfugiés auprès du HCR.

Le nombre de personnes répertoriées commença alors logiquement à augmenter à partir de l'année 2004, conséquence de l'enlisement de l'armée américaine face à la résistance irakienne. Un nouveau palier fut franchi en 2006 suite à la destruction d'un haut lieu du chiisme à Samara par la guérilla sunnite et le basculement de l'Irak dans la guerre confessionnelle (Al-Khalidi \& Tanner, 2009). Ainsi, fin 2007, certains rapports ${ }^{11}$ estimaient à deux millions le nombre des Irakiens qui s'étaient déplacés à l'intérieur de leur pays, alors que 2 autres millions auraient fui leur patrie pour se réfugier chez leurs voisins arabes comme la Jordanie et la Syrie surtout ${ }^{12}$. Les autorités syriennes donnèrent le chiffre de 1,4 millions d'Irakiens qui auraient trouvé refuge dans leur pays sur la période 2003-2007. Au plus fort du conflit, 30000 à 45000 personnes seraient entrées dans le pays tous les mois, selon la même source (Dagge, 2007). Même s'il s'agit d'estimations, ces chiffres sont demeurés sujet à caution, étant donné, pour chacun des protagonistes, l'usage politique qu'il serait possible d'en faire. Susceptibles d'être 
"gonflés» ou à l'inverse minimisés, nous les citons à titre indicatif et non pour relancer un débat qui n'a plus lieu d'être. Cette période est caractérisée par une grande tolérance des autorités syriennes à l'égard des Irakiens qui se présentaient aux frontières du pays. Dès 2003, la Syrie a pratiqué une politique d'ouverture de sa frontière pour les Irakiens. Considérés simplement comme des ressortissants arabes ${ }^{13}$, ces derniers ont disposé d'un visa de 3 mois délivré à la frontière, et ce jusqu'au $1^{\mathrm{er}}$ semestre 2007. La Syrie est ainsi devenue le premier pays d'accueil des réfugiés irakiens. La carte que nous présentons en figure 3, construite sur des estimations, donne une image de l'importance du phénomène pour cette période.

Figure 3 : Les Irakiens hors d'Irak au Moyen-Orient en 2008

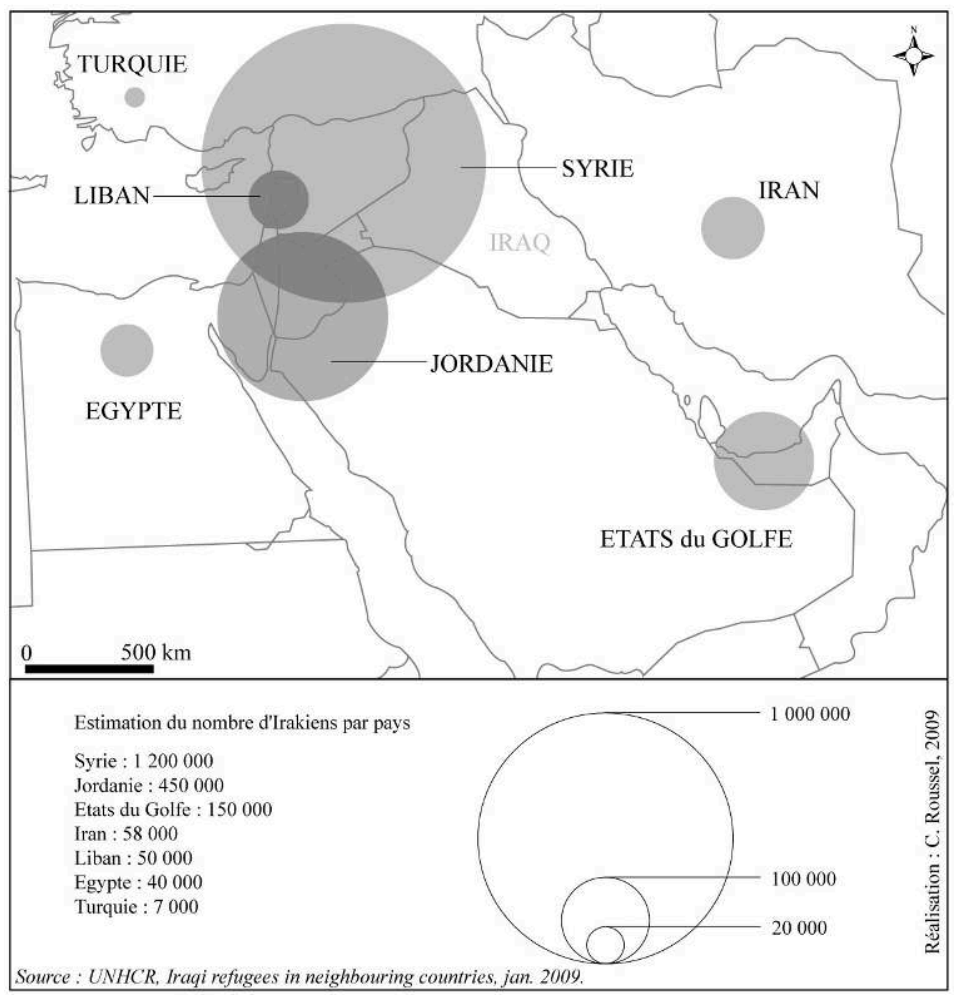

Le $1^{\text {er }}$ octobre 2007, des mesures de restriction sur l'entrée des Irakiens en Syrie a entraîné une modification profonde des comportements migratoires autant pour ceux présents sur le territoire syrien que pour ceux désireux de le rejoindre. Dorénavant, avec l'obligation d'obtenir un visa avant l'entrée en Syrie, l'accès au territoire syrien est devenu plus compliqué pour les Irakiens. Une rupture nette s'est donc produite à cette date dans la politique syrienne d'accueil des Irakiens d'autant que les conditions d'obtention du visa sont strictes.

Les conséquences de la mise en place du visa pour les Irakiens furent rapides: des retours massifs; un ralentissement du nombre d'entrées en Syrie des Irakiens; une réduction du nombre d'allers-retours ${ }^{14}$. Mais surtout, comme le note justement $\mathrm{M}$. $\mathrm{K}$. Doraï (2009), «de nombreuses personnes se sont retrouvées de facto en situation d'illégalité à expiration de leur visa de tourisme ». Cela a abouti à la genèse "d'un groupe de personnes résidentes de fait en Syrie, mais dans l'impossibilité de légaliser leur situation », devenant du même coup des migrants illégaux. Et lorsque l'on sait que seules « certaines catégories de personnes » [irakiennes] ont le droit à une carte de 
séjour temporaire d'un an, "comme les parents d'enfants scolarisés en Syrie ou les personnes suivant un traitement médical et leur famille» (Doraï, 2009), cette disposition laisse envisager un avenir bien difficile pour les Irakiens réfugiés en Syrie.

\section{B. Installation provisoire ou durable des réfugiés irakiens à Jeramana?}

Nombre de rapports sur les réfugiés se rejoignent sur le fait que les communautés minoritaires irakiennes chrétiennes et sabéennes, sont menacées de disparition sur leur sol nata ${ }^{15}$. Si depuis 2008 , beaucoup d'Irakiens sont rentrés au pays comme nous venons de le voir, les réfugiés issus des petites minorités confessionnelles demeurent cantonnés dans l'exil. Pour eux, pas question de rentrer dans leur pays d'origine compte tenu de la forte discrimination qu'ils ont subie et que leurs coreligionnaires continuent à subir en Irak. Ces groupes confessionnels, faibles démographiquement par rapport au triptyque chiite/sunnite/kurde ${ }^{16}$, n'ont pas leurs propres milices en $\operatorname{Irak}^{17}$ et sont physiquement menacés. Ils doivent compter, pour leur protection, sur la police qui est souvent corrompue et même parfois l'auteur direct de violences ethniques et sectaires. La plupart d'entre eux ont vu une partie de leur famille se faire décimer, kidnapper et ne possèdent plus aucun bien en Irak. Leurs maisons ou fonds de commerce, par exemple, ont généralement été vendus pour financer leur départ et subvenir quelques temps à leurs besoins élémentaires ${ }^{18}$.

Les Chrétiens et Sabéens venus d'Irak, que l'on retrouve à Jeramana, cherchent généralement à poursuivre leur parcours migratoire en Europe, en Amérique du Nord ou en Australie. Pour eux, la Syrie est pensée avant tout comme un espace de transit et d'attente avant une réinstallation ultérieure. Il est certain que pour beaucoup cette représentation d'un avenir meilleur dans un pays occidental risque de s'avérer bien chimérique car, pour la majorité, l'attente se prolonge dans le refuge syrien. C'est dans l'optique d'un départ vers des pays tiers, que l'enregistrement auprès du HCR s'effectue, car il permet d'opérer une demande d'asile ou de regroupement familial. Cependant les effectifs acceptés dans les pays riches demeurent largement en dessous des demandes effectuées et ne concernent normalement que les personnes les plus vulnérables ${ }^{19}$. Ainsi, les réseaux familiaux ou communautaires - associations caritatives religieuses ; instances religieuses présentes dans les pays occidentaux - sont-ils souvent mobilisés pour aider les candidats au départ à poursuivre vers des pays hôtes. Ces réseaux transnationaux expliquent que les minoritaires ont plus de facilités que les autres Irakiens pour trouver des chemins de sortie et ainsi poursuivre leur parcours migratoire vers un pays tiers (Daniş, 2006). L'exemple de la famille de Mahâ ${ }^{20}$, jeune fille sabéenne de Bagdad, est sur ce point éclairant :

Au début de l'année 2000, Mahâ et sa sœur ont obtenu un visa de tourisme pour la France à Dubaï. En mars, elles se rendirent en France. Leur père, au cours de la même année, parvint, grâce à une invitation de son frère qui est directeur d'un hôtel en France, à obtenir lui-aussi un visa; il rejoignit ses filles. En 2003, ils reçoivent leur carte de séjour. Leur mère et leur frère restèrent à Bagdad jusqu'en 2004, date de leur sortie. Une demande de regroupement familial effectuée en 2003 n'ayant pas aboutie, toute la famille décida de se retrouver en Syrie à Jeramana. En été 2004, Mahâ et sa mère se présentèrent à l'ambassade de France à Damas. Le consul accorda alors à sa mère un visa pour la France. Après un retour précipité à Bagdad, la mère de Mahâ quitta la Syrie pour la France début 2005. Seul le frère resta à Jeramana; d'âge adulte, il ne pouvait bénéficier du regroupement familial. 
Inscrit comme réfugié au HCR, il est parvenu à quitter la Syrie en 2008 dans le cadre d'un programme de réinstallation de réfugiés vers les Etats-Unis. En Irak, il avait travaillé de nombreuses années dans une compagnie américaine comme agent de sécurité et avait conservé des liens avec des ressortissants américains influents.

Enfin, certains ont recours à des filières clandestines pour se rendre en Europe. Des passeurs tentent de vendre de faux passeports aux Irakiens, documents généralement issus de trafics avec certains pays de l'est de l'Europe comme la Bulgarie. Ces passeports sont généralement proposés avec des billets d'avion pour des sommes parfois astronomiques (de 10 à $20000 \$$ selon les témoignages et selon les cas). En fait, le voyage n'est pas un aller direct vers une capitale européenne, ce qui éveillerait trop facilement les soupçons des douaniers. L'itinéraire proposé passe généralement par l'Asie (Chine, Indonésie, Malaisie) là où les contrôles apparaissent comme peu scrupuleux avant de se terminer en France ou en Italie. De là, si le clandestin n'est pas appréhendé en route, il tentera de rejoindre généralement la Suède par la route.

Le cas de Jeramana ne peut pas être généralisé à l'ensemble des lieux-réceptacles des réfugiés irakiens de la région damascène. Le fait que cette ville ait reçu une grande part des Irakiens issue des minorités confessionnelles explique que beaucoup de réfugiés y sont restés par manque d'alternatives. Il faut donc tenir compte de la très forte hétérogénéité des migrants irakiens (réalités différentes en fonction des moyens financiers et de l'identité religieuse) pour appréhender les notions d'espace de transit et d'espace-refuge dans le cas de la migration irakienne en Syrie.

\section{Le visage de Jeramana depuis l'arrivée des réfugiés irakiens}

29 Facteurs de mutations spatiales et économiques indéniables à l'échelle de la ville, les réfugiés irakiens à Jeramana se sont caractérisés, à leur arrivée, par une dynamique d'investissement propre (apport personnel d'argent). Mais avec un conflit irakien qui s'installe dans la durée, les sources de financement - généralement des économies - se tarissent. Alors les irakiens réfugiés en Syrie basculent de plus en plus nombreux dans la précarité, l'informel, la débrouille.

\section{A. La répartition spatiale des réfugiés irakiens en Syrie : une concentration dans les périphéries de l'agglomération de Damas}

Les transformations de certaines villes de la banlieue damascène peuvent-être - comme l'exemple de Jeramana le montre - concomitantes aux secousses géopolitiques qui secouent le Moyen-Orient, provoquant, telle une onde de choc, des mouvements de populations. A Jeramana, les conflits israélo-arabes de 1948 et de 1967 avaient déjà déclenché deux vagues de réfugiés palestiniens, créant et renforçant le camp de Jeramana à l'entrée principale de la ville. En 1967, une partie des déplacés du Golan qui fuyaient l'occupation israélienne sont venus trouver refuge dans la région damascène : Jeramana fut un des lieux principaux d'implantation.

31 Les ressortissants irakiens, comme les autres populations réfugiées, se sont répartis entre les diverses grandes villes syriennes avec une forte concentration dans la région urbaine de Damas (voir figure 4). Des groupes de moindre importance sont à noter à Alep, Homs et Hassakeh. Ces réfugiés, surtout d'origine urbaine - la plupart originaires 
de Bagdad $^{21}$ - sont venus s'insérer dans le tissu urbain syrien. Dans ce pays, pas de camps de réfugiés comme pour les Palestiniens au Liban voisin, les Afghans autour de Peshawar ou les réfugiés du Darfour ${ }^{22}$, et donc aucune image de flots de populations dépourvues lancées sur les routes de l'exil. Il s'est agi d'un « écoulement » certes massif de population mais qui a généralement eu le temps de s'organiser, de préparer son départ mais aussi son lieu d'arrivée (location d'appartements ou achat pour les plus aisés, stratégie de regroupement familial auto-organisé, entraide et solidarité à l'intérieur des réseaux de famille ou de connaissance - colocation, services divers...). Ainsi, d'après les entretiens que nous avons menés, rares furent les Irakiens à fuir leur pays sans un point de chute en perspective. Ceci corrobore bien évidemment notre analyse précédente concernant le regroupement communautaire et la vigueur des réseaux familiaux et confessionnels.

Figure 4 : Distribution des réfugiés irakiens inscrits au HCR par gouvernorat syrien en 2009

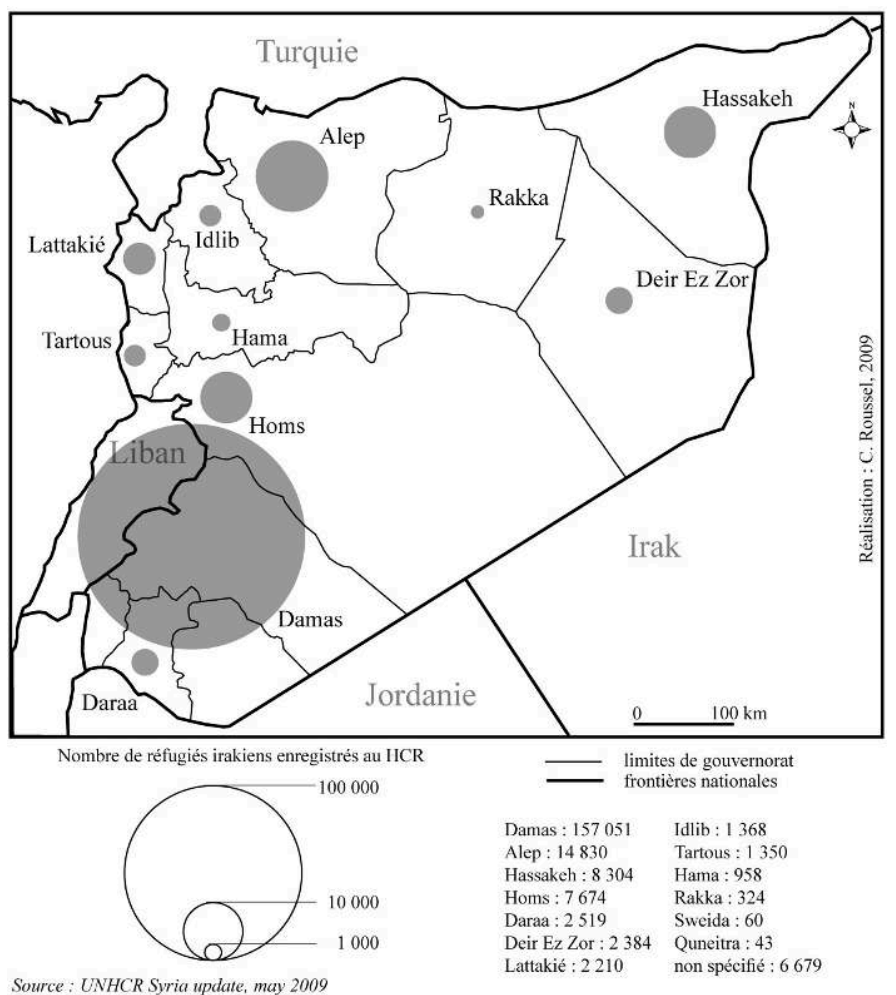

Jeramana apparaît comme le premier pôle d'implantation des réfugiés irakiens (enregistrés auprès du HCR) en Syrie. La carte (figure 5) montre la répartition des réfugiés dans le tissu urbain de la région damascène. Jeramana et Sayyida Zeynab semblent concentrer à elles seules plus de $50 \%$ des réfugiés irakiens de l'agglomération damascène, ce que les études de terrain confirment. Sayyida Zeynab, lieu de pèlerinage et pôle migratoire pour des groupes chiites de tous horizons, ressemble dans son processus de peuplement à celui de Jeramana. 


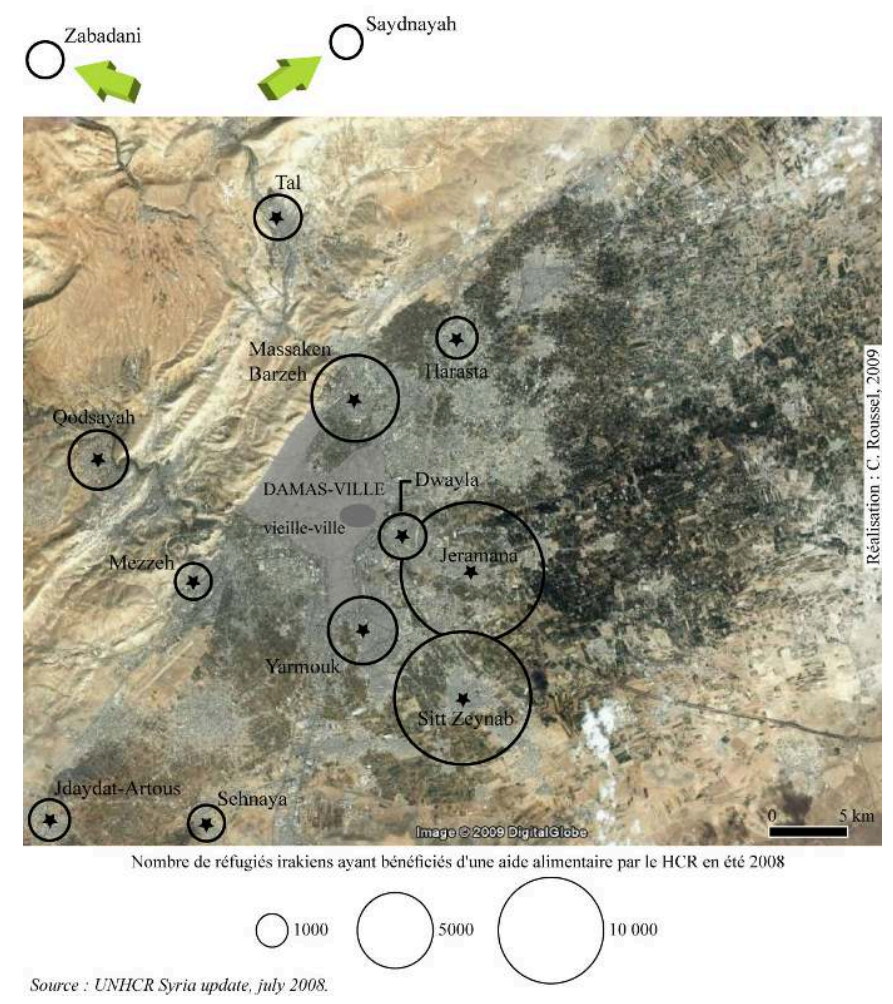

\section{B. Une crise passagère de l'immobilier}

33 L'arrivée des Irakiens à Jeramana a entraîné, jusqu'à la fin 2007, une pénurie de logements. Si les premiers Irakiens à quitter le pays étaient des gens plutôt aisés qui trouvaient facilement à se loger dans les quartiers résidentiels de Damas, à partir de 2006 surtout $^{23}$, on vit arriver des personnes beaucoup moins aisées avec très peu d'économies qui s'entassaient à plusieurs familles dans un même appartement Rapport de la DDC, Direction du Développement et de la Coopération Suisse (2007). Selon le même rapport, « d'après une étude du HCR en Syrie, 93 \% des réfugiés irakiens louaient leur appartement en 2006 et leur loyer était souvent majoré par rapport à leurs congénères syriens". Cette forte demande en logements a provoqué une importante inflation sur le marché de l'immobilier : les prix ont triplé parfois voire quadruplé, comme à Jeramana.

Un appartement de $2 / 3$ pièces qui se louait habituellement autour des 5000 LS avant l'afflux des Irakiens trouvait preneur à 20000 LS. La forte demande favorisa les excès : beaucoup de logements furent divisés pour être proposés à la location sous forme de pièce unique. Les garages, sous-sols et autres pièces aveugles se louèrent à prix d'or à des familles parfois nombreuses dans la détresse. «Les derniers arrivants ont de plus en plus de peine à trouver un toit et leurs conditions d'hébergement se détériorent (appartements surpeuplés, alors que d'autres vivent dans des caves froides et humides ou dans des garages sans fenêtre, ni eau courante) » indique le rapport de la DDC Suisse (2007).

L'inflation toucha également le secteur commercial. Les fonds de commerce pouvaient se vendre jusqu'à cinq fois leur valeur de départ en fonction de l'emplacement. 
Rapidement, le gouvernement prit des mesures pour interdire la vente des fonds de commerce aux Irakiens. Seule la location resta donc autorisée même si des moyens de contourner l'interdiction de délivrer des certificats de propriété aux Irakiens existent bien. Beaucoup d'Irakiens tentent logiquement de survivre en Syrie grâce à une activité commerciale : à Jeramana, on ne compte plus les épiceries irakiennes, les agences de taxi pour l'Irak et les restaurants irakiens.

Cette forte demande a modifié en quelques années la physionomie de la ville de Jeramana. Des immeubles en béton de cinq ou six étages ont remplacé les anciennes constructions basses des années 70 et 80 qui étaient pour la plupart des lieux d'habitation. Lors de la décennie écoulée, elles furent tour à tour démolies. S'y substituent des constructions où le rez-de-chaussée est dorénavant consacré aux locaux commerciaux, les étages, aux appartements à louer. La photographie de la figure $\mathrm{n}^{\circ} 1$ en fournit un exemple probant. L'élévation des bâtiments ne s'est pas produite uniquement dans le centre ville, mais s'est diffusée jusque dans les quartiers périphériques.

Depuis le second semestre 2008, la situation du foncier s'est améliorée avec le retour d'une partie des Irakiens dans leur pays. La pression est retombée et même si les appartements restent chers à la location, l'offre était, fin 2008, plus vaste et variée que les années précédentes.

\section{Précarité des réfugiés et développement du travail informel}

Nous avons noté précédemment que le départ vers la Syrie s'était effectué, pour beaucoup d'Irakiens, à l'aide de leur épargne ou/et des revenus issus de la vente de leurs biens en Irak (maison, commerce...), tout du moins pour les plus chanceux d'entre eux. Mais avec des loyers élevés et les augmentations spectaculaires des prix des produits de consommation courante - énergie, alimentation - les économies des réfugiés se sont rapidement amenuisées. C'est alors que les difficultés commencèrent pour beaucoup. C'est d'ailleurs une des raisons principales du retour d'une partie des réfugiés irakiens dans leur pays d'origine. Avec le durcissement du droit de séjour en Syrie, l'assèchement des ressources financières apparait, selon une enquête du HCR, comme la raison majeure du retour des Irakiens, bien avant une quelconque amélioration de la situation sécuritaire en Irak.

Le manque de moyens financiers est rarement compensé par une activité professionnelle rémunérée qui permettrait à tous les membres d'une même famille de survivre en Syrie avec un salaire. Même ce salaire permet rarement de payer le loyer mensuel du logement. Hormis les Irakiens les moins dépendants financièrement - à Jeramana ce sont les petits entrepreneurs et certains commerçants -, la plupart des Irakiens tentent d'exercer une activité professionnelle dans un marché de l'emploi largement saturé. Dans la ville de Jeramana, beaucoup sont employés de manière informelle dans les innombrables ateliers qui se sont développés dans les sous-sols des immeubles pour produire en toute discrétion chaussures, vêtements, bijoux, boites de mouchoirs en papier et autres biens manufacturés. Les salaires perçus y dépassent rarement les 6000 LS (100 euros) par mois. Le petit commerce ambulant s'est dans ces conditions largement développé dans les rues de la ville. Le soir surtout, de jeunes gens proposent aux passants cartes téléphoniques, allumettes et cigarettes, etc. 
La précarisation des réfugiés irakiens en Syrie apparaît sous divers aspects :

- un problème de subsistance, car la plupart des Irakiens arrivés en Syrie n'ont pas de quoi subvenir à leurs besoins sur le long terme. Ils vivent sur leurs économies comme on vient de le noter.

- certains adultes ou enfants travaillent au noir pour des salaires de misère, qui représentent souvent le seul revenu familial.

- hormis les personnes possédant une carte de séjour, les autres doivent composer avec l'absence de statut légal qui caractérise leur condition. Ainsi, seules des activités salariales dans le secteur informel leur sont ouvertes, ce qui renforce d'autant plus leur situation de précarité.

\section{Les Irakiens dans la ville : développement du secteur commercial}

La présence irakienne se remarque d'abord par le nombre d'enseignes commerciales qui font référence à l'Irak. Les restaurants proposent des plats irakiens comme le masgou $f^{24}$; ils sont particulièrement nombreux dans deux secteurs de la ville : à l'entrée de Jeramana - en venant de Damas avant la place du président - et à la sortie de la ville par l'autoroute de l'aéroport. Dans ces deux zones, les emplacements étaient libres lors de l'arrivée des Irakiens (période 2004-2006). A l'inverse, les commerciaux irakiens sont peu présents dans la rue principale de Jeramana (ashara el ham); ce secteur est tenu depuis longtemps par des Druzes originaires de la ville ou du Djebel el-Arab (gouvernorat de Sweida). Par contre, nombreux sont les épiciers irakiens à tenir de petites boutiques ou un atelier de confection/réparation dans les rues adjacentes de la ville. Une multitude de commerces tenus par des Irakiens se sont ainsi ouverts à Jeramana depuis 2005. Ils sont généralement en liaison avec leur pays d'origine qui reste en général le lieu d'écoulement de leur marchandise. Ils produisent donc à Jeramana des biens destinés au marché irakien.

Depuis la fermeture du garage des taxis pour l'Irak, suite à des affrontements entre Druzes syriens et Irakiens, les clients en partance pour les villes irakiennes sont pris directement dans les nombreuses agences qui se situent également à l'entrée de la ville, avant la place du président. L'activité de transport de marchandises ou de passagers se déroule le soir car les voyages vers l'Irak s'effectuent généralement de nuit.

A la sortie de la ville, avant l'autoroute pour l'aéroport, un espace de loisir s'est développé, destiné à la clientèle irakienne chrétienne pour l'essentiel. La consommation d'alcool se fait dans des restaurants qui cuisinent des plats traditionnels irakiens ou dans des boites de nuit avoisinantes qui diffusent les hits des chanteurs irakiens. Cet espace a trouvé sa place sur des terrains inoccupés comme des jardins ou des terrains vagues, alors que tout ce secteur était autrefois dévolu uniquement aux activités mécaniques (garages de réparation d'automobiles, centres de nettoyage, stations d'essence). Ces activités « salissantes » côtoient aujourd'hui ce nouveau lieu de loisir destiné à la détente des Irakiens de Jeramana.

\section{Conclusion}

Au terme de cet article, nous conclurons sur deux points : la dynamique d'organisation socio-spatiale de l'agglomération damascène et le rôle d'une ville comme Jeramana dans le champ migratoire des Irakiens de confession chrétienne et sabéenne. 
L'installation, au sein de l'agglomération de Damas, de populations migrantes (syriens de l'exode rural, réfugiés irakiens) s'est produite dans des espaces déjà marqués par la présence de migrants et déjà fortement homogènes du point de vue confessionnel. Le cas de Jeramana, qui apparaît certes comme un exemple emblématique de cette dynamique socio-spatiale, n'est cependant pas isolé. De plus en plus, des quartiers damascènes et même des villes voisines de la Ghouta, constitutives de la couronne urbaine de ce qu'il convient d'appeler dorénavant le Grand Damas, deviennent des territoires confessionnels dans lesquels s'exprime une appartenance identitaire affirmée. C'est précisément cela qui génère une atmosphère urbaine propre à une ville comme Jeramana. Ainsi cette caractéristique - être une centralité pour des migrants issus de minorités confessionnelles - renforce son rôle de territoire communautaire au sein de l'aire urbaine damascène, espace avec lequel elle reste en grande partie déconnectée au point de vue des pratiques socio-spatiales de ses habitants. Car l'étude de leurs pratiques permet de remarquer que les habitants de Jeramana sont reliés systématiquement aux territoires d'origines de leur groupe respectif : Druzes du Sud au gouvernorat de Sweida; Chrétiens syriens avec leur ville d'origine; réfugiés irakiens avec l'Irak. Ainsi, les transports en commun au départ de Jeramana desservent-ils Sweida (bus pullman); les trois lignes de microbus au départ de Jeramana desservent Bab Touma (quartier chrétien), le garage de Qaboun (pullman pour les villes du nord, lieux d'origine de nombreux chrétiens comme Homs) et Baramké (site universitaire et hub du réseau de transport de la capitale). Une multitude d'officines tenues par des Irakiens desservent de nombreuses villes d'Irak à l'aide de taxis-services. Par contre, aucune ligne de bus/minibus ne la relie aux villes voisines toutes peuplées de musulmans sunnites: Mleiha, Aqraba... Cette dynamique d'occupation et de pratique sélective de l'espace reposant sur une organisation communautaire de la société qui la produit, apparaît, selon nous, comme un élément majeur de la différenciation spatiale en œuvre dans la capitale et ses banlieues.

Si la ville de Jeramana s'est avérée, dans le contexte actuel, comme un lieu d'implantation pérenne pour des dizaines de milliers de réfugiés irakiens, c'est que ces derniers se retrouvent pour beaucoup dans un « cul-de-sac ».L'originalité de Jeramana tient dans le fait que les réfugiés irakiens qui s'y sont installés appartiennent à des groupes confessionnels persécutés dans leur pays précisément pour leur identité religieuse. Ces minoritaires, dont une forte proportion demeure bloquée en ce lieu d'exil, sont plongés dans l'attente d'une solution alternative au retour. Pour beaucoup, le provisoire devient durable; le lieu de transit, envisagé à l'origine comme tel dans l'élaboration des projets migratoires, se pérennise. Cette réalité ne doit pas masquer les tentatives des réfugiés irakiens pour rejoindre un pays d'accueil et surtout l'espoir d'y parvenir un jour. Même si peu y parviennent, les réfugiés irakiens en attente de réinstallation dans un pays tiers perçoivent d'abord Jeramana comme un relais dans un champ migratoire plus vaste. Il n'en demeure donc pas moins que la migration irakienne vers la Syrie conserve sa dynamique interne, ce qui ne l'empêche pas de venir se connecter à des centralités migratoires plus anciennes, propres elles-aussi à d'autres groupes de migrants, comme à Jeramana. Il n'est donc pas surprenant de constater que ces «strates" de populations, culturellement distinctes, conservent une capacité propre d'insertion dans la ville, génèrent leurs propres espaces économiques et de loisir, et enfin déploient leurs propres stratégies de survie. 


\section{BIBLIOGRAPHIE}

Al-Khalidi A., Tanner V., 2009, «L'Irak remodelé par l'épuration confessionnelle », in L'Etat du Monde, Edition 2009, pp.287-293.

Chatelard G., Doraï M.K., 2009, « La présence irakienne en Syrie et en Jordanie : dynamiques sociales et spatiales, et modes de gestion par les pays d'accueil », Maghreb-Machrek, n¹99, pp. 43-60.

Dagge J., 2007, « Accueil des réfugiés irakiens en Syrie », http://www.unv.org/fr/nos-activites/ pays/republique-arabe-syrienne/doc/accueil-des-refugies-irakiens.html, 28 juin 2008.

Daniș D., 2006, « « Attendre au Purgatoire » : Les réseaux religieux de migrants chrétiens d'Irak en transit à Istanbul », Revue Européenne des Migrations Européennes, vol.33, n³, pp.109-134.

Direction du développement et de la coopération suisse DDC, 2007, Reportage en Jordanie et en Syrie, http://www.ddc.admin.ch/fr/Home_21719/Item_154490/Item_154544, 03 février 2008.

Doraï M.K., 2009, « L'exil irakien à Damas. Modes d'insertion urbaine et reconfiguration des réseaux migratoires », EchoGéo, nº 8, http://echogeo.revues.org/index10976.html, 4 avril 2009.

Kawakibi S., 2008, «La migration irrégulière en Syrie. Les réfugiés irakiens comme cas d'étude », Carim notes d'analyse et de synthèse, $\mathrm{n}^{\circ}$ 66, Centre Robert Schuman, European University Institute, 9

La Civita M.J.L., 2008, Les chrétiens d'Irak pris en otage, http://www.cnewacanada.ca/mag-bodypop-ca-fr.aspx?articleID=3279, 13 mars 2009.

Minority rights group international MRG, 2008, Iraq, World Directory of Minorities and Indigenous Peoples, http://www.minorityrights.org/6741/iraq/liraq-franais.html, 14 février 2008.

UNHCR, 2009, Syria Briefing Notes (May 2009).

UNHCR, 2009, Syria-Statistical Report on registered Iraqis (August 2008 ; August 2009).

UNHCR, 2009, Syria update (July 2008 ; september 2009).Rapports et sites :

UNHCR, http://www.unhcr.org/cgi-bin/texis/vtx/page?page=49e486426

\section{NOTES}

1. Cf. l'article de Fabrice Balanche dans ce numéro.

2. Je le montre dans ma thèse en m'appuyant sur l'étude des migrations internes en provenance des provinces du Sud syrien.

3. Ce travail s'inscrit dans un programme de recherche financé par l'ANR et intitulé Tanmia, «Le Développement: Fabrique de l'action publique dans le monde arabe ». Nous tenons à remercier les initiateurs de ce programme sans qui le travail de terrain n'aurait pu se faire.

4. La commune de Jeramana s'étend sur 600 hectares. Environ 450 sont voués aux constructions, le reste étant des jardins. Sur la totalité de la surface bâtie, les constructions illégales s'étendent sur près 120 hectares.

5. 114363 selon le recensement de 2004 .

6. Le nombre d'inscrits au HCR sous-estime les effectifs réels car tous les réfugiés ne viennent pas se faire enregistrer. 
7. La mohafaza de Quneitra, dont une partie est occupée depuis 1967 par Israël, s'est dépeuplée d'une grande partie de sa population. Presque la moitié de la population de cette région s'est installée à Damas et dans sa région périurbaine.

8. Dans cette montagne vit une petite communauté druze de quelques milliers d'âmes, réparties entre une douzaine de villages.

9. Les Sabéens ou Mandéens sont les adeptes de St Jean-Baptiste qui baptisa Jésus.

10. Les Nations Unies rapportent que le tiers de toutes les personnes déplacées en Irak sont chrétiennes.

11. Rapport des Nations Unies de juin 2007 ; rapport de la coopération allemande de septembre 2007.

12. Dans une moindre mesure, l'Iran (environ 50 000), l'Égypte (100 000), le Liban (de 20000 à 40 000) et d'autres États du Golfe.

13. Même régime d'entrée et de séjour très souple que les autres ressortissants arabes. Les Irakiens ne sont pas considérés par les autorités syriennes comme des réfugiés mais sont soumis à la législation et la réglementation sur l'entrée et le séjour des étrangers. Durant cette période, les Irakiens étaient considérés comme des touristes.

14. Utiliser avant le $1^{\mathrm{er}}$ octobre 2007 surtout pour renouveler le visa de tourisme puisqu'une simple sortie et une entrée le même jour permettait d'obtenir un nouveau visa de 3 mois.

15. «Les Sabiens-Mandéens, qui pratiquent une ancienne croyance gnostique, sont menacés d'extinction en tant que peuple. Environ $80 \%$ de la population a été expulsée ou tuée depuis 2003. D'après le témoignage du dirigeant de l'Union des Associations Mandéennes devant l'US CIRF au mois de juillet, il reste seulement 5000 adhérents en Iraq. Des centaines de meurtres, d'enlèvements et d'incidents de torture ont souvent été accompagnés d'une rhétorique accusant les Sabiens de sorcellerie, d'impureté et d'adultère systématique. Les femmes sabiennesmandéennes furent cibles de viol et de mort pour avoir refusé de se voiler ». Extrait d'un rapport en ligne sur le site de l'ONG Minority rights group international (2008).

16. Les réfugiés irakiens chiites et les sunnites en Syrie, plus nombreux en Irak et donc à même d'assurer leur protection dans ce pays, sont rentrés plus massivement au pays.

17. «Les chrétiens d'Irak n'ont aucune protection, poursuit-il. Les musulmans arabes, les Kurdes, les Shiites ou les Sunnites d'Irak bénéficient de la protection de leurs tribus. Si un membre de leur famille est tué, les autres membres de la famille le vengent » (LA CIVITA, 2008).

18. Pire encore, "certaines familles ont révélé (sous l'anonymat pour protéger les membres de leur famille encore en Irak) qu'elles avaient été forcées, sous la menace des fusils de la milice, de céder leurs maisons et leurs commerces, avec tout leur contenu » (LA CIVITA, 2008).

19. Entre février 2007 et la fin du mois d'août 2009, 14098 Irakiens ont été réinstallés dans un pays d'accueil dont près de 10000 aux USA. Viennent loin derrière, le Canada, l'Allemagne et l'Australie. Source : UNHCR Syrie, September 2009.

20. Entretien réalisé en avril 2009 à Paris. Mâha était étudiante à l'Inalco Paris durant l'année 2008-2009. Elle était une de mes étudiantes.

21. $67 \%$ des réfugiés inscrits auprès de l'organisation onusienne en 2009 (source : UNHCR Syria, Statistical Report on registered Iraqis, 31 Auguste 2009).

22. Cf. l'article de Philippe Quintin dans ce numéro.

23. 2006 coïncide avec le début des affrontements inter-confessionnels en Irak et les vagues d'attentats destinées à terroriser la population.

24. Carpe grillée au feu de bois. 


\section{RÉSUMÉS}

Alimentées par des flux migratoires multiformes, les banlieues des villes du Proche-Orient connaissent un développement urbain soutenu et une croissance démographique élevée. A partir de l'exemple de Jeramana, nous analyserons les types de flux migratoires qui convergent vers cet important pôle d'implantation et les raisons qui poussent les migrants à privilégier ce lieu au détriment d'un autre. Dans le contexte de conflit armé en Irak qui perdure, cet article accorde une place centrale à l'étude des réfugiés irakiens en Syrie et à leur capacité à transformer l'espace urbain des périphéries du Grand Damas.

Supplied with multiform migratory flows, the suburbs of the cities of the Middle East know a steady urban development and a high population growth. From the example of Jeramana, we will analyze the types of migratory flows which converge towards this important pole of settlement and the reasons that make migrants privilege this place to the detriment of another one. In the context of perdurable armed conflict in Iraq, this article grants a central place to the study of the Iraqi refugees in Syria and to their capacity to transform the urban space of the outskirts of Greater Damascus.

\section{INDEX}

Keywords : community, Damascus, development, iraqi refugee, migration, Syria, urban transformation

Mots-clés : communauté, Damas, développement, migration, mutation urbaine, réfugié irakien, Syrie

\section{AUTEUR}

\section{CYRIL ROUSSEL}

Géographe, chercheur associé au Gremmo de Lyon - UMR 5195 GREMMO -

cyril.roussel@netcourrier.com 